\title{
Utilization Patterns of Glucagon-Like Peptide-1 Receptor Agonists in Patients with Type 2 Diabetes Mellitus in Italy: A Retrospective Cohort Study
}

\author{
Marco Orsini Federici · Janette McQuillan • Giovanni Biricolti • \\ Serena Losi · Jeremie Lebrec • Catrina Richards - Cristiana Miglio • \\ Kirsi Norrbacka
}

Received: December 19, 2017 / Published online: March 10, 2018

(C) The Author(s) 2018. This article is an open access publication

\begin{abstract}
Introduction: Real-world evidence on glucagon-like peptide-1 receptor agonist (GLP-1 RAs) usage is emerging in different European countries but is lacking in Italy. This retrospective cohort study aimed to describe the real-world drug utilization patterns in patients initiating GLP-1 RAs for treating T2DM in Italy.
\end{abstract}

Enhanced content To view enhanced content for this article go to https://doi.org/10.6084/m9.figshare. 5924998.

Electronic supplementary material The online version of this article (https://doi.org/10.1007/s13300018-0396-2) contains supplementary material, which is available to authorized users.

M. O. Federici · S. Losi

Eli Lilly SPA, Via A. Gramsci, 731-733, 50019 Sesto

Fiorentino, FI, Italy

J. McQuillan · C. Richards · C. Miglio

QuintileIMS, 210 Pentonville Road, London N19JY, UK

G. Biricolti

Eli Lilly SpA, Via Thailandia, 27, 00144 Rome, Italy

J. Lebrec

Eli Lilly Deutschland GmbH, Werner-Reimers-Straße 2-4, 61352 Bad Homburg, Germany

K. Norrbacka $(\varangle)$

Eli Lilly Finland, Laajalahdentie 23, 00330 Helsinki, Finland

e-mail: norrbacka_kirsi@lilly.com
Methods: Adults aged $\geq 20$ years and with $\geq 1$ oral antidiabetic drug (alone or in combination with insulin) other than GLP-1 RAs in the 6 months prior to initiating exenatide twice daily (exBID), exenatide once weekly (exQW), dulaglutide once weekly (DULA), liraglutide once daily (LIRA) or lixisenatide once daily (LIXI) between March and July 2016 were retrospectively identified in the Italian IMS LifeLink $^{\mathrm{TM}}$ longitudinal prescriptions database (retail pharmacy data). Patients with $\geq 6$ month follow-up (defined as evidence of any prescription activity) were included. Proportions of patients who remained persistent (continued treatment until discontinuation/ switch) in the first 6 months and of those who discontinued or switched to a different GLP-1 RA over the entire follow-up were recorded. For each treatment, the average daily/weekly dosage (ADD/AWD) while persistent during the available follow-up was calculated.

Results: We identified 7319 patients: 92 exBID, 970 exQW, 3368 DULA, 2573 LIRA and 316 LIXI. Across treatments, $89 \%$ patients were $\geq 50$ years old, $54 \%$ were males, and the median follow-up duration ranged between 8.1 and 8.7 months. At 6 months, 35\% exBID, 47\% exQW, 62\% DULA, $50 \%$ LIRA and $40 \%$ LIXI patients remained persistent. Over the entire follow-up, median persistence days varied from 73 (exBID) to $>300$ days (DULA). The mean $\pm \mathrm{SD} \mathrm{ADD} /$ AWD was exBID: $17.7 \pm 2.1 \mu \mathrm{g} /$ day; exQW: 
$2.1 \pm 0.1 \mathrm{mg} /$ week; DULA: $1.5 \pm 0.2 \mathrm{mg} /$ week; LIRA: $1.5 \pm 0.2 \mathrm{mg} /$ day; LIXI: $21.0 \pm 5.5 \mu \mathrm{g} /$ day. Conclusions: This real-world analysis suggests differences exist in persistence between patients treated with various GLP-1 RAs. Among the investigated treatments, patients prescribed exBID recorded the lowest and those prescribed DULA the highest persistence with therapy.

Funding: Eli Lilly and Co., Indianapolis, IN, USA.

Keywords: Glucagon-like peptide-1 receptor agonists (GLP-1 RAs); Persistence; Prescribed average daily dose; Treatment modifications; Type two diabetes

\section{INTRODUCTION}

In Italy, 3.5 million people have been diagnosed with diabetes, over $90 \%$ of whom have type 2 diabetes mellitus (T2DM), with an estimated burden for the Italian national health system of approximately $€ 8-10$ billion yearly for drugs, hospitalizations and visits $[1,2]$.

Despite recent advances that have expanded the available therapeutic options, achieving and maintaining glycemic control in patients with T2DM remains a major challenge for doctors who must face complex therapeutic decisions [3]. The 2016 Italian Standard of Care [4], in line with the updated 2015 joint position statement released by the American Diabetes Association and European Association for the Study of Diabetes [5], recommends a patient-centered approach with metformin as the first line of therapy and the addition of one (or two) of the other available treatments as the second (or third) line of therapy, with no indicated preference. Doctors should make their initial decision based on the patient's physical and clinical characteristics and the efficacy and safety profiles of the available treatments and then address the eventual progressive worsening of glycemic control through treatment intensification.

Glucagon like peptide-1 receptor agonists (GLP-1 RAs) are a relatively new class of injectable drugs that have emerged as an attractive second- or third-line therapeutic option because of their association with improved glycemic control, lower hypoglycemia rate and weight loss, although gastrointestinal GLP-1 RAs side effects have also been reported $[6,7]$. There are several GLP-1 RAs on the market in Europe, each with specific characteristics [3]; they vary in the magnitude of their effect in reducing HbA1c and enhancing weight loss and also in their adverse event profiles [3, 8]. Additionally, injection frequencies are variable, with some GLP-1 RAs offering more convenient dosing (weekly vs. daily injections) and potential higher adherence [9] compared with others. In Italy, the currently available GLP-1 RAs include exenatide twice daily (from now on referred to as exBID), launched February 2008; liraglutide once daily (LIRA), launched August 2010; exenatide once weekly (exQW) and lixisenatide once daily (LIXI), both launched December 2013 and dulaglutide (DULA) once weekly launched February 2016.

For a drug to be effective, patients must adhere to and persist with therapy. Previous studies have shown an association between better adherence and persistence with glucoselowering treatments and improved clinical and economic diabetes-related outcomes [10-13]. Persistence with GLP-1 RAs has also been reported to be associated with positive clinical outcomes and reduced healthcare costs $[14,15]$. However, to date, real-world data on persistence, discontinuation or switch dynamics with GLP-1 RA are limited. Only a few studies have compared treatment patterns or variable dosing across the different treatments $[9,15-20]$, and none of them were conducted in Italy.

Given the current limited evidence and the important clinical and costs implications, the main objective of this study was to provide realworld evidence on treatment patterns for T2DM GLP-1 RA therapy initiators in Italy, specifically persistence with the GLP-1 RA (i.e., index therapy) and treatment modifications, including discontinuation, switch to another hyperglycemic therapy, dosing changes and augmentation (addition of new therapy to the index treatment). Other objectives included evaluating the average daily/weekly dose (ADD/ AWD) of the index therapy and describing the 
baseline characteristics of the patients initiating the different GLP1-RA treatments. These outcomes were retrospectively evaluated using the Italian Longitudinal Prescription (LRx) database, a large database containing information on retail dispensing.

\section{METHODS}

This study was a retrospective cohort study, including all patients receiving the first prescription of GLP-1 RA as exBID, exQW, DULA, LIRA or LIXI. To allow for comparisons, the overall study design and methodologies were like those used in previous studies conducted in other European countries [16, 17]. Ethics committee approval is not required for secondary use of pseudonymized prescription data in Italy.

\section{Data Source}

The Italian LRx database (QuintileIMS, Durham, NC, and Danbury, CT, USA) accesses nationwide pharmacy data centers processing prescription data of all products reimbursed by the National Health System (class "A" products) or those reimbursed under specific circumstances, as per guidelines issued by the Italian Medicines Agency (class "A_PHT" products). Pharmacies are required to report details of these prescriptions to the Italian Ministry of Health to receive reimbursement. LRx covers $90 \%$ of prescriptions in the retail channel (weighted percentage between 90\% and 73\%) in Italy. Drugs dispensed in the hospital are not captured within LRx. However, hospital dispensation is expected to account only for a minority $(<10 \%)$ of the overall GLP-1 RA units sold in Italy (IQVIA MIDAS $^{\circledR}$ data; not shown).

Data are entered at the point of sale from retail pharmacies based on prescriptions that have been dispensed. Data are then uploaded from sites to a vendor where they are collated and further pseudonymized (de-identified).

Patient and prescription details in LRx include: sex and 5-year age band and prescription data, including EphMRA Anatomical Classification (ATC) code, quantity dispensed, prescriber specialty and date of dispensation. Diagnoses are not recorded in LRx.

\section{Study Design and Population Selection}

An overview of the study design is provided in Fig. 1. The study period was from 24 August 2015 (6 months prior to the most recently launched GLP-1 RA in Italy, DULA) to 31 January 2017 (last available data at the time of analyses). The index date was the date of first prescription for any of the GLP-1 RAs of interest (exBID, exQW, DULA, LIRA or LIXI) and the initiated therapy was termed the index treatment. Patients were included in the study if they had a 6-month continuous eligibility period (based on evidence of any prescription activity within the database) prior to and after the index date for baseline characterization and outcome evaluation. Therefore, the eligible index date was between 24 February 2016 and 30 July 2016. Patients were required to have received at least one prescription for other antidiabetic drugs, including oral treatments alone or in combination with insulins (proxy for T2DM diagnosis) and no GLP-1 RA treatment in the 6 months prior to the index date (look-back period). All the available follow-up from the index date until the earliest of the end of study period or end of continuous eligibility period were used for outcome evaluation, resulting in variable follow-up durations with a minimum of 6 months for each patient.

Only adult patients were analyzed in line with the GLP-1 RA indication. As age is only recorded in the database in 5-year bands, patients aged $\geq 20$ years at the start of GLP-1 therapy were selected. Once selected, patients were assigned to one of the five study cohorts, based on the index treatment received: exBID, exQW, DULA, LIRA or LIXI cohorts. Patients prescribed liraglutide indicated for weight management $\left(\right.$ Saxenda ${ }^{\circledR}$ ) were excluded from the analyses.

\section{Measures and Analyses}

Baseline characteristics were described for all patients and by treatment cohort and included 


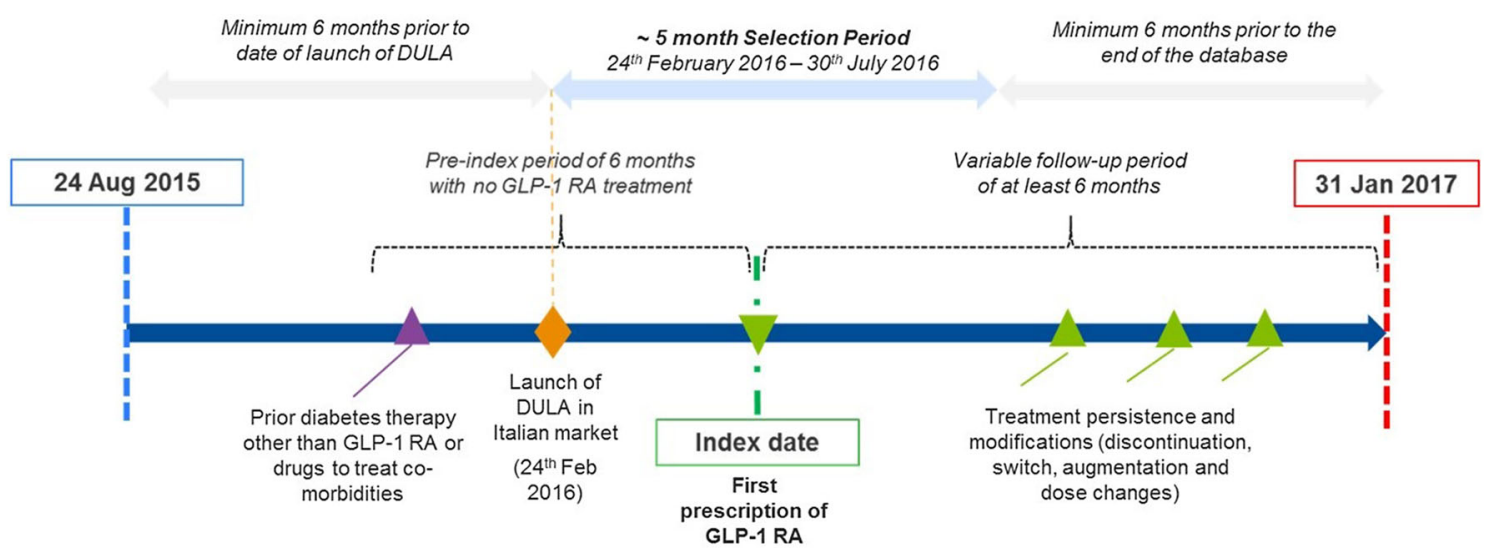

Fig. 1 Overview of study design. DULA dulaglutide, GLP-1 RA glucagon-like peptide-1 receptor agonist

age band at the time of GLP-1 RA treatment initiation, gender and record of any antihyperglycemic therapy class other than GLP-1 RA (and the number of classes used) or other drugs to treat comorbidities prescribed in the 6 months prior to the index date. Other antihyperglycemic therapy classes of interest were oral anti-diabetics or insulins, including alpha glucosidase inhibitors, biguanide (i.e., metformin), dipeptidyl peptidase (DPP)-4 inhibitors, meglitinides, sodium-glucose cotransporter (SGLT)-2 inhibitors, sulfonylurea, thiazolidinediones, fixed oral combinations, short-acting insulins, basal insulins and premixed insulins. Treatments for comorbidities included antidepressants, anti-emetics, weightloss medication (including anti-obesity medications apart from Saxenda), anti-platelet medication, cardiovascular (CV) medication, antihypertensive drugs, anti-arrhythmic drugs, lipid-lowering agents and non-steroidal antiinflammatory drugs (NSAIDs).

For all patients and by study cohort, the mean and standard deviation (SD) duration of follow-up in days was calculated. The resulting duration was divided by 30 days to obtain the follow-up duration in months (irrespective of calendar months). Over this time, the proportions and percentage of patients who experienced treatment modifications, including discontinuation, switch, dose changes and augmentation of therapy were evaluated. In addition, persistence was evaluated at the fixed 6-month minimum follow-up in terms of persistence proportions (and 95\% CI) and over the variable follow-up period in terms of probability of remaining persistent over time and median (and 95\% CI) persistence duration (days), using the Kaplan-Meier method.

Patients were considered persistent (i.e., on continued index therapy) until evidence of discontinuation or switch. Discontinuation was defined as the occurrence of a gap in a series of successive prescriptions that was $>2$ times the expected drug lasting time as per prescription. The day after the expected drug lasting time as per prescription end was defined as the date of discontinuation. A repeat prescription of the patients' index medication after this gap period was considered a restart and was not classified as continuation of the index therapy. A prescription of a new non-index therapy (including other GLP-1 RAs) within 30 days prior to or following a discontinuation was considered a change of therapy (switch). The date of the new non-index prescription was defined as the date of switch. Treatment augmentation was defined as a new non-index antihyperglycemic prescription (other than GLP-1 RAs), started over 30 days prior to the end of follow-up or the index discontinuation date. Dose changes included down- or up-titration, defined as any dose decrease or increase of the index therapy. If the dose increase was within the recommended licensed dose as indicated in the summaries of product characteristics [21], then the dose change was defined as on-label up-titration; if the dose increase was beyond the licensed 
doses, then it was defined as off-label uptitration.

The ADD of the index therapy was assessed for all patients over the available follow-up duration while on continued index treatment (i.e., while persistent), irrespective of any dose change or augmentation of therapy. Daily dose was calculated by dividing the total amount or units of drug prescribed by the number of days between two consecutive prescriptions. ADD was initially evaluated by calendar month intervals for patients with an index therapy prescription within that month. Average ADDs over calendar months were summarized to provide an overall ADD. An average weekly dose (AWD) was calculated for exQW and DULA by multiplying the daily dose by 7 . More details on the calculation of ADD can be found in Divino et al. [17].

All analyses performed in this study were descriptive, and no statistical tests were performed to formally compare the treatment cohorts. This article does not contain any new studies with human or animal subjects performed by any of the authors.

\section{RESULTS}

\section{Selected Population and Baseline Characteristics}

The population attrition is shown in Figure $\mathrm{S} 1$ in the supplementary material.

A total of 7319 patients met the inclusion criteria and were analyzed for their patient characteristics (Table 1), treatment utilization (Table 2) and average daily doses (Table 3 ). The majority were males (54\%), and $89 \%$ were aged 50 years or older. Of the selected population, $1 \%(N=92)$ of patients initiated treatment with exBID, $13 \% \quad(N=970) \quad$ with exQW, $46 \%$ $(N=3368)$ with DULA, 35\% $(N=2573)$ with LIRA and $4 \%(N=316)$ with LIXI. While the age distribution was similar across the different treatment cohorts, there was a higher proportion of females who started treatment with exBID (60\%) compared with other GLP-1 RA therapies (44-50\%).
Overall, 78\% patients were on metformin therapy in the 6 months prior to initiating GLP1 RA treatment, with similar proportions across the different treatment cohorts (76-84\%); 36\% were on monotherapy (30-50\% across the different cohorts), $35 \%$ on dual therapy (26-39\% across the different cohorts), and $27 \%$ used three or more therapy classes $(13-43 \%$ across the different cohorts). The most commonly used other non-antidiabetic treatments were for cardiovascular diseases (86\% overall, $85-90 \%$ across different treatment cohorts), antihypertensive drugs $(78 \%, 75-85 \%)$ and lipid-lowering medication (54\%, 52-62\%).

\section{Treatment Persistence and Modifications}

The overall mean (SD) follow-up duration was similar across the different cohorts and was 8.3 (1.4) months, minimum 6.0 and maximum 11.3 months.

Although descriptive analysis was done, the results suggest that treatment patterns differed between treatment groups.

Treatment persistence at 6 months, duration of persistence and treatment modifications over the available follow-up are shown in Table 2 . Across all treatments, almost half of the patients on any GLP-1 RA remained persistent with their initial therapy (i.e., they did not discontinue or change the index treatment) at 6 months (54\%; $95 \%$ CI 53, 56). This proportion was higher for patients on DULA $(62 \% ; 95 \%$ CI 60,64$)$ and lower for those on exBID (35\%; 95\% CI 25,45$)$ and LIXI $(40 \%$; 95\% CI 35, 45) compared with exQW (47\%; 95\% CI 43, 50) and LIRA (50\%; 48, 52). Over the full follow-up period, median persistence time $(95 \% \mathrm{CI})$ varied from 73 days $(62,140)$ for patients on exBID to 183 days $(167$, 203) for patients on LIRA, while the median was not reached for patients on DULA (i.e., median time to end of persistence $>300$ days) (Fig. 2). The fact that patients starting treatment with DULA had the highest probability of remaining persistent with the index therapy compared with other GLP-1 RAs at and beyond 6 months is illustrated by the Kaplan-Meier curves (Fig. 2). The highest risk of discontinuing or switching 
Table 1 Baseline patient characteristics

\begin{tabular}{lcccccc}
\hline & All study GLP-1 RAs & exBID & exQW & DULA & LIRA & LIXI \\
\hline Patients, $n$ (\%) & $7319(100)$ & $92(1.3)$ & $970(13.3)$ & $3368(46.0)$ & $2573(35.2)$ & $316(4.3)$ \\
Age at index year, $n$ (\%) & & & & & & \\
20-29 & $17(0.2)$ & $0(0.0)$ & $2(0.2)$ & $8(0.2)$ & $7(0.3)$ & $0(0.0)$ \\
$30-39$ & $120(1.6)$ & $0(0.0)$ & $15(1.6)$ & $61(1.8)$ & $36(1.4)$ & $8(2.5)$ \\
$40-49$ & $682(9.3)$ & $7(7.6)$ & $99(10.2)$ & $320(9.5)$ & $224(8.7)$ & $32(10.1)$ \\
50-64 & $3344(45.7)$ & $34(37.0)$ & $466(48.0)$ & $1536(45.6)$ & $1168(45.4)$ & $140(44.3)$ \\
$\geq 65$ & $3156(43.1)$ & $51(55.4)$ & $388(40.0)$ & $1443(42.8)$ & $1138(44.2)$ & $136(43.0)$ \\
Gender, $n$ (\%) & & & & & \\
Female & $3338(45.6)$ & $55(59.8)$ & $432(44.5)$ & $1474(43.8)$ & $1218(47.3)$ & $159(50.3)$ \\
Male & $3981(54.4)$ & $37(40.2)$ & $538(55.5)$ & $1894(56.2)$ & $1355(52.7)$ & $157(49.7)$ \\
Prior antidiabetic therapy & other than GLP-1 RA, $n(\%)^{\mathrm{a}}$ & & & & \\
None & $156(2.1)$ & $3(3.2)$ & $34(3.5)$ & $52(1.5)$ & $65(2.5)$ & $2(0.6)$ \\
AGI & $364(4.9)$ & $4(4.3)$ & $45(4.6)$ & $148(4.3)$ & $149(5.7)$ & $18(5.6)$ \\
Metformin & $5713(78.0)$ & $77(83.6)$ & $761(78.4)$ & $2556(75.8)$ & $2056(79.9)$ & $263(83.2)$ \\
DPP-4 inhibitors & $2010(27.4)$ & $4(4.3)$ & $242(24.9)$ & $1205(35.7)$ & $489(19.0)$ & $70(22.1)$ \\
Fixed oral combinations & $2228(30.4)$ & $9(9.7)$ & $273(28.1)$ & $1273(37.7)$ & $602(23.3)$ & $71(22.4)$ \\
Meglitinides & $645(8.8)$ & $10(10.8)$ & $80(8.2)$ & $279(8.2)$ & $238(9.2)$ & $38(12.0)$ \\
Basal insulins & $1354(18.4)$ & $13(14.1)$ & $98(10.1)$ & $382(11.3)$ & $734(28.5)$ & $127(40.1)$ \\
Short-acting insulins & $603(8.2)$ & $4(4.3)$ & $50(5.1)$ & $172(5.1)$ & $325(12.6)$ & $52(16.4)$ \\
Pre-mix insulins & $44(0.6)$ & $0(0.0)$ & $6(0.6)$ & $15(0.4)$ & $18(0.6)$ & $5(1.5)$ \\
SGLT-2 inhibitors & $397(5.4)$ & $5(5.4)$ & $53(5.4)$ & $213(6.3)$ & $117(4.5)$ & $9(2.8)$ \\
Sulfonylurea & $2213(30.2)$ & $25(27.1)$ & $306(31.5)$ & $1100(32.6)$ & $684(26.5)$ & $98(31.0)$ \\
Glitazones & $835(11.4)$ & $6(6.5)$ & $120(12.3)$ & $440(13.0)$ & $240(9.3)$ & $29(9.1)$
\end{tabular}

Number of prior antidiabetic therapy classes used (other than GLP-1 RAs), $n$ (\%)

\begin{tabular}{lcccccc}
0 & $156(2.1)$ & $3(3.2)$ & $34(3.5)$ & $52(1.5)$ & $65(2.5)$ & $2(0.6)$ \\
1 & $2626(35.8)$ & $46(50)$ & $381(39.2)$ & $1155(34.2)$ & $950(36.9)$ & $94(29.7)$ \\
2 & $2573(35.1)$ & $31(33.6)$ & $344(35.4)$ & $1330(39.4)$ & $785(30.5)$ & $83(26.2)$ \\
$3+$ & $1964(26.8)$ & $12(13.0)$ & $211(21.8)$ & $831(24.7)$ & $773(30.0)$ & $137(43.4)$ \\
Other prior therapy, $n(\%)^{\mathrm{b}}$ & & & & & & \\
Antidepressants & $857(11.7)$ & $15(16.3)$ & $115(11.8)$ & $349(10.3)$ & $343(13.3)$ & $35(11.0)$ \\
Anti-emetics & $8(0.1)$ & $0(0.0)$ & $0(0.0)$ & $2(0.0)$ & $5(0.1)$ & $1(0.3)$ \\
Weight-loss medication & $0(0.0)$ & $0(0.0)$ & $0(0.0)$ & $0(0.0)$ & $0(0.0)$ & $0(0.0)$ \\
\hline
\end{tabular}


Table 1 continued

\begin{tabular}{lcccccc}
\hline & All study GLP-1 RAs & exBID & exQW & DULA & LIRA & LIXI \\
\hline Anti-platelet medication & $2493(34.0)$ & $36(39.1)$ & $332(34.2)$ & $1062(31.5)$ & $939(36.4)$ & $124(39.2)$ \\
CVD medication & $6321(86.3)$ & $83(90.2)$ & $823(84.8)$ & $2877(85.4)$ & $2261(87.8)$ & $277(87.6)$ \\
Antihypertensives & $5683(77.6)$ & $78(84.7)$ & $731(75.3)$ & $2553(75.8)$ & $2060(80.0)$ & $261(82.5)$ \\
Antiarrhythmics & $154(2.1)$ & $2(2.1)$ & $18(1.8)$ & $56(1.6)$ & $68(2.6)$ & $10(3.1)$ \\
Lipid-lowering drugs & $3977(54.3)$ & $57(61.9)$ & $502(51.7)$ & $1814(53.8)$ & $1423(55.3)$ & $181(57.2)$ \\
NSAIDs & $1911(26.1)$ & $26(28.2)$ & $270(27.8)$ & $869(25.8)$ & $666(25.8)$ & $80(25.3)$ \\
\hline
\end{tabular}

exBID exenatide twice daily, exQW exenatide once weekly, DULA dulaglutide, LIRA liraglutide, LIXI lixisenatide. AGI alpha glucosidase inhibitors, DPP dipeptidyl peptidase, $S G L T$ sodium-glucose co-transporter, $C V D$ cardiovascular disease, NSAIDs non-steroidal anti-inflammatory drugs

a Patients on fixed combinations were considered to be on two different drug classes

b Prescribed in the 6 months prior to the index date (i.e., start of GLP-1 RA treatment)

therapy was recorded in the first 2 months of treatment (Fig. 2).

The occurrence of treatment modifications also varied among the different treatment cohorts (Table 2). Over the available follow-up, $63 \%$ of patients on exQW and $66 \%$ of those on DULA experienced at least one treatment change compared with $79 \%$ on exBID and $73 \%$ on LIRA. The highest proportion of patients with at least one treatment modification was recorded for patients on LIXI (86\%).

The exBID cohort recorded the highest proportion of patients whose first treatment change was a discontinuation (60\%), while those on DULA recorded the lowest (32\%). Patients on exQW did not experience dose changes as first treatment modification. Across other treatments, $5-10 \%$ of patients experienced a dose increase as a first treatment change, while 7-12\% experienced a dose increase at any time. Switch (5-9\%) and augmentation (5-6\%) proportions were similar across the different treatment cohorts.

\section{Average Daily/Weekly Doses}

According to the summary of product characteristics of the different GLP-1 RAs [22-24], the recommended initial dose of exBID is $5 \mu \mathrm{g}$ twice daily, increased to $10 \mu \mathrm{g}$ twice daily after
1 month; LIRA should be initiated with a dose of $0.6 \mathrm{mg}$ once per day, which can be increased to $1.2 \mathrm{mg}$ after at least a week. Doses can be further increased to $1.8 \mathrm{mg}$ after at least another week on $1.2 \mathrm{mg}$ for non-responsive patients; LIXI is also administered once per day, with a starting dose in the 2 weeks following initiation of $10 \mu \mathrm{g}$ and with a fixed maintenance dose of $20 \mu \mathrm{g}$ on day 15 and thereafter; exQW $(2 \mathrm{mg})$ and DULA $(0.75 \mathrm{mg}$ as monotherapy or $1.5 \mathrm{mg}$ in combination with other drugs) are administered once per week. The overall average daily and weekly dose over the available follow-up period recorded in this study is shown in Table 3 for all patients and by treatment cohort. For all treatments but LIXI, the prescribed ADD/ AWD was in the ranges of the licensed doses. For LIXI, a slightly higher ADD of $21.0 \mu \mathrm{g}$ was recorded.

\section{DISCUSSION}

The results of this retrospective analysis of Italian prescribing data show that there are differences in the real-world utilization patterns of different GLP-1 RAs. Previous real-world studies in Italy have examined diabetes-related outcomes related to GLP-1 RAs [25-27]; this is the first published study evaluating the real-world 
Table 2 Persistence with the index therapy and treatment modifications over the available follow-up duration

\begin{tabular}{|c|c|c|c|c|c|c|}
\hline & $\begin{array}{l}\text { All study GLP-1 } \\
\text { RAs }\end{array}$ & exBID & exQW & DULA $^{a}$ & LIRA & LIXI \\
\hline Patients, $n(\%)$ & $7319(100)$ & $92(1.3)$ & $970(13.3)$ & $3368(46)$ & $2573(35.2)$ & $316(4.3)$ \\
\hline \multicolumn{7}{|c|}{ Persistence, i.e., no discontinuation or switch of the index treatment } \\
\hline At 6 months, $N(\%)$ & $3984(54.4)$ & $32(34.8)$ & $451(46.5)$ & $\begin{array}{l}2084 \\
(61.9)\end{array}$ & $1291(50.2)$ & $126(39.9)$ \\
\hline $\begin{array}{l}95 \% \text { CI of } \\
\text { proportions }\end{array}$ & $53.3,55.6$ & $25.1,44.5$ & $43.4,49.6$ & $60.2,63.5$ & $48.2,52.1$ & $34.5,45.3$ \\
\hline \multicolumn{7}{|c|}{ Persistence duration (days between the index date and end of follow-up) } \\
\hline Median $(95 \% \mathrm{CI})$ & $238(219,293)$ & $\begin{array}{c}73(62 \\
140)\end{array}$ & $\begin{array}{c}150(128 \\
176)\end{array}$ & $>300^{\mathrm{a}}$ & $\begin{array}{c}183(167 \\
203)\end{array}$ & $\begin{array}{c}113(88 \\
151)\end{array}$ \\
\hline 25th percentile & 62 & 62 & 58 & 86 & 63 & 51 \\
\hline \multicolumn{7}{|c|}{ At least a first index treatment modification (including dose changes) } \\
\hline$N(\%)$ & $5073(69.3)$ & $73(79.3)$ & $610(62.9)$ & $\begin{array}{l}2231 \\
(66.2)\end{array}$ & $1886(73.3)$ & $273(86.4)$ \\
\hline \multicolumn{7}{|c|}{ First treatment modification type, $N(\%)^{\mathrm{b}}$} \\
\hline On-label up-titration & $305(4.2)$ & $4(4.3)$ & $0(0.0)$ & $161(4.8)$ & $140(5.4)$ & $0(0.0)$ \\
\hline Off-label up-titration & $178(2.4)$ & $1(1.1)$ & $0(0.0)$ & $89(2.6)$ & $57(2.2)$ & $31(9.8)$ \\
\hline Down-titration & $791(10.8)$ & $2(2.2)$ & $0(0.0)$ & $531(15.8)$ & $214(8.3)$ & $44(13.9)$ \\
\hline Augmentation & $437(6.0)$ & $5(5.4)$ & $57(5.9)$ & $197(5.8)$ & $162(6.3)$ & $16(5.1)$ \\
\hline Discontinuation & $2956(40.4)$ & $55(59.8)$ & $497(51.2)$ & $\begin{array}{l}1086 \\
(32.2)\end{array}$ & $1164(45.2)$ & $154(48.7)$ \\
\hline Switch & $406(5.5)$ & $6(6.5)$ & $56(5.8)$ & $167(5.0)$ & $149(5.8)$ & $28(8.9)$ \\
\hline \multicolumn{7}{|c|}{ Up-titration at any time by type, $N(\%)^{c}$} \\
\hline On-label up-titration & $340(4.6)$ & $5(5.4)$ & $0(0.0)$ & $178(5.3)$ & $157(6.1)$ & $0(0.0)$ \\
\hline Off-label up-titration & $247(3.4)$ & $1(1.1)$ & $0(0.0)$ & $126(3.7)$ & $81(3.1)$ & $39(12.3)$ \\
\hline
\end{tabular}

exBID exenatide twice daily, exQW exenatide once weekly, DULA dulaglutide, LIRA liraglutide, LIXI lixisenatide

${ }^{a}$ Median persistence days for this cohort could not be calculated as more than $50 \%$ of the patients, over the entire followup, remained persistent

$\mathrm{b}$ Percentages are calculated over the total number of patients, overall and within each treatment cohorts

${ }^{c}$ Up-titration at any time was defined as any two consecutive prescriptions exceeding the index dose

treatment patterns of individual GLP-1 RAs in Italy. The results add to the evidence base coming from other European countries as the number of GLP-1 RAs available on the European market increases.
Based on our findings, patients initiating treatment with DULA are more likely to remain persistent compared with other drugs, while greater proportions of patients initiating on exBID and LIXI tend to discontinue or switch treatment. Across the different treatments, 
Table 3 Overall average daily and weekly doses by treatment cohort

\begin{tabular}{llllll}
\hline & exBID & exQW & DULA & LIRA & LIXI \\
\hline Number of patients included in the analyses $^{\mathrm{a}}$ & 48 & 687 & 2742 & 1854 & 204 \\
Average daily dose while persistent & $\mathrm{mcg}$ & $\mathrm{mg}$ & $\mathrm{mg}$ & $\mathrm{mg}$ & $\mathrm{mcg}$ \\
Mean (SD) & $17.66(2.11)$ & $0.30(0.02)$ & $0.22(0.03)$ & $1.54(0.22)$ & $21.01(5.45)$ \\
Median & 16.55 & 0.30 & 0.22 & 1.49 & 19.34 \\
Average weekly dose while persistent & & $\mathrm{mg}$ & $\mathrm{mg}$ & & \\
Mean (SD) & - & $2.12(0.14)$ & $1.52(0.19)$ & - & - \\
Median & - & 2.08 & 1.52 & - & - \\
\hline
\end{tabular}

exBID exenatide twice daily, exQW exenatide once weekly, DULA dulaglutide, LIRA liraglutide, LIXI lixisenatide, $S D$ standard deviation (estimated neglecting repeated use of some patients), $m g$ milligrams, $m c g$ micrograms

a Average doses were calculated for patients who remained persistent with the index therapy and had at least two consecutive prescriptions for the index therapy

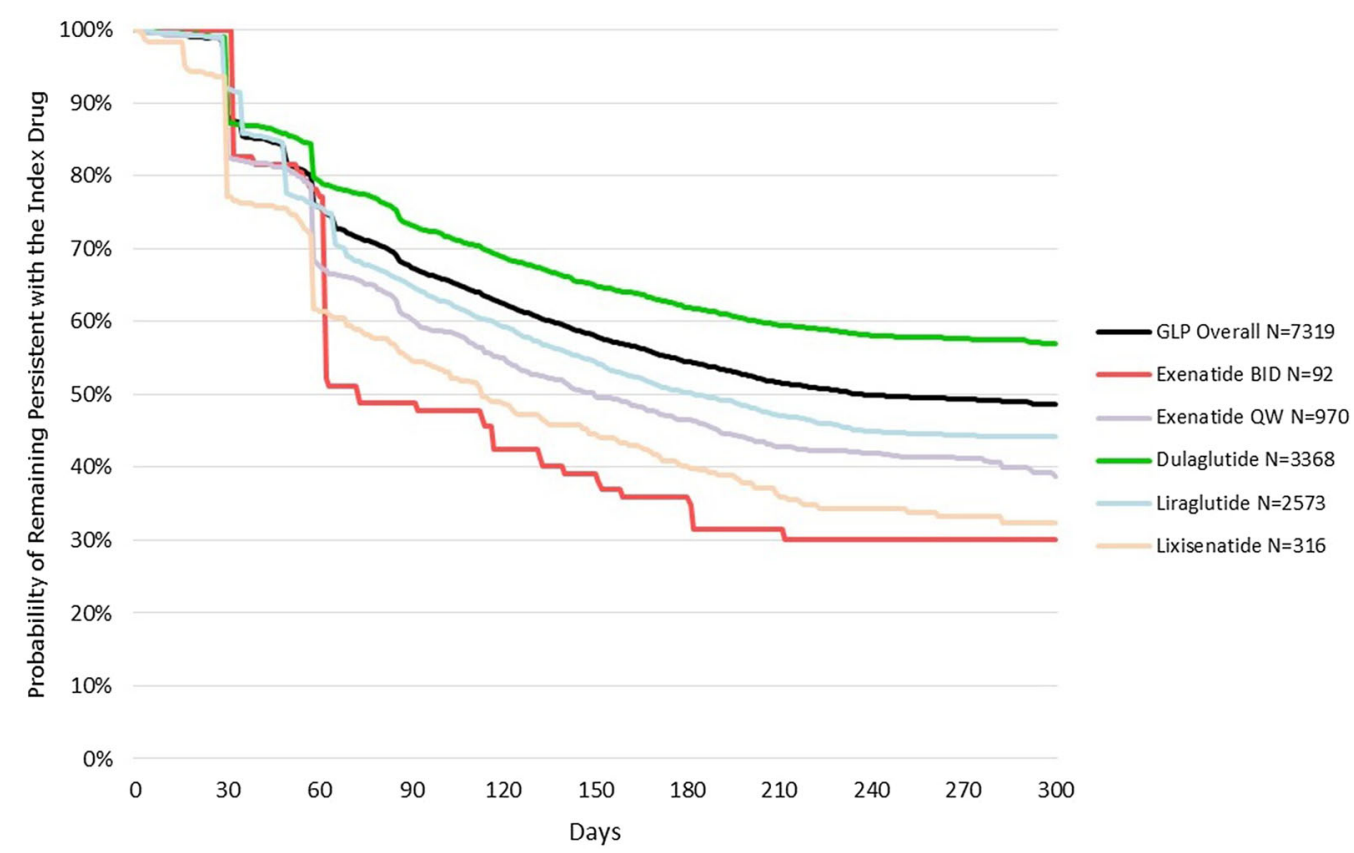

Fig. 2 Kaplan-Meier analyses over the available follow-up durations: probability of remaining persistent with the index therapy. exBID exenatide twice daily, exQW exenatide once weekly, DULA dulaglutide, LIRA liraglutide, LIXI lixisenatide

persistence with the index therapy at 6 months was $\quad$ exBID $<$ LIXI $<$ exQW $<$ LIRA $<$ DULA. The KM analysis over the full follow-up period confirmed this trend and showed that patients initiating on DULA are less likely to discontinue or switch therapy than patients on other treatments. There are many possible reasons for low treatment persistence, including high injection frequency (twice daily vs. once daily vs. weekly injections), patient low tolerability of treatment, adverse reactions or lack of effectiveness. On the other hand, a higher persistence with therapy has been reported to result in better glycemic control and economic outcomes, with 
an estimated reduced healthcare cost of $27-30 \%$ $[14,15]$. Although not ascertained as part of this study, therapies with improved persistence could be associated with better outcomes and lower costs.

The differences in treatment patterns observed in this study are consistent with those recorded in other European countries and in the US. In a similar analysis conducted in T2DM initiating GLP-1 RA treatment in 2013, Divino and colleagues [16] examined treatment patterns with exBID, exQW, LIRA and LIXI over 1 year from initiation in five European countries. The proportion of persistent patients was lower for exBID compared with LIRA and exQW in Germany ( $29 \%$ vs. $43 \%$ and $33 \%$, respectively), The Netherlands (34\% vs. $61 \%$ and $51 \%$ ) and Sweden (31\% vs. $59 \%$ and $43 \%$ ). The proportion of persistent patients was also lower for exBID in Belgium compared with LIRA and LIXI (18\% vs. $29 \%$ and $50 \%$ ) and in France compared with LIRA ( $44 \%$ vs. 52\%). Previously, the same authors [17] reported that persistence was lower for patients newly initiating on exBID (47-74\% across Germany, Belgium, The Netherlands, UK and Sweden) compared with LIRA (51-80\%) and exQW (58-75\%) in the first 6 months of treatment. Alatorre and colleagues [9] looked at treatment patterns of patients initiating GLP-1 RA treatment with DULA vs. exQW and LIRA between November 2014 and April 2015 in the US. At 6 months from initiation, the proportion of patients discontinuing treatment was lower for DULA vs. exQW (26\% vs. $48 \%)$ and for DULA versus LIRA ( $28 \%$ vs. $36 \%$ ). While these trends are overall in line with the results of this study, different proportions across countries can be explained by the inherent characteristics of the local health systems as well as differences in the study designs, time when the studies were carried out and follow-up durations.

This study also found that the average daily doses prescribed to patients on the different GLP-1 RA treatments were generally within the recommended ranges, but for LIXI this was slightly above the recommended range (21.0 $\mathrm{mg}$ recorded in this study, label range 5-10 mg twice daily), suggesting that some patients on this drug may require higher doses to maintain or achieve glycemic control.
Differences in the methods used to calculate ADD may limit comparisons with other studies. The methodology used in this study was similar to that used by Divino and colleagues [16], who recorded an ADD of $1.55 \mathrm{mg}$ for patients on LIRA in Germany, similar to this study. ADD varied in other countries and was lower in Belgium $(1.41 \mathrm{mg})$ and higher in France $(1.64 \mathrm{mg})$, The Netherlands $(1.68 \mathrm{mg})$ and Sweden $(1.60 \mathrm{mg})$. In the same study, the authors also recorded an ADD of $20.1 \mathrm{mg}$ for patients on LIXI in Germany [16], in line with the current study, and confirm that slightly higher doses than expected for this treatment may be prescribed by doctors in the real world.

The authors acknowledge some limitations to the study design. First of all, we were unable to investigate reasons for treatment modifications (lack of effectiveness, adverse events, etc.) as these clinical details are not covered by prescription databases. Second, selection bias toward more severe patients may have occurred because of our continuous enrollment requirements based on prescribing activity. However, this effect should be minor in patients affected by chronic diseases, such as diabetes, who are regularly taking treatments over long period of times. In addition, the continuous enrollment criterion was necessary to ensure adequate visibility into the patients' clinical history. Third, some of the GLP-1 RAs have been launched in Italy only recently; therefore, the average follow-up duration was short $(<1$ year). Additional research is needed to confirm our results over longer time periods and to support clinically relevant findings with statistical testing. Finally, irrespective of the launch date of the different products in Italy, the sample size varied greatly across the different treatment cohorts, with only 92 patients identified who initiated treatment with exBID. This small sample size calls for caution in the interpretation of the exBID results.

Limitations consistent with a pharmacybased database should also be taken into account when interpreting the results of this study. Patients who purchase prescriptions outside the pharmacies included in the database will not have that prescription utilization recorded in the database, which may result in 
an underestimation of the drug usage. Doses were calculated according to the dispensed packs over time, which means that stockpiling of prescriptions or filling prior to prescription run-out could lead to overestimations in dose. Furthermore, data are only collected from pharmacies that participate in the database, which may cause some levels of selection bias. However, the impact of this is considered to be very limited in the Italian LRx, as the database coverage is around $90 \%$ on average across all regions. Finally, pharmacy data do not record patient mortality. Consequently, patients may be considered discontinued because of no evidence of prescriptions after the minimum 6-month follow-up period rather than dead.

\section{CONCLUSIONS}

This study adds to the limited literature on type 2 diabetes mellitus and GLP-1 receptor agonists by providing new evidence on the real-world usage of exenatide (twice daily and once weekly), dulaglutide, liraglutide and lixisenatide in Italy. Despite country-specific differences in databases, prescribing and dispensing practices and the analytical methods used, the results of the current analysis are aligned with those from other countries and indicate that patients on exenatide twice daily are less likely to remain persistent with their therapy than those on other GLP-1 RA treatments with either weekly (exenatide QW and dulaglutide) or daily dosing schedules (liraglutide and lixisenatide). Patients with the highest persistence initiated dulaglutide. In addition, for lixisenatide, the average daily dispensed doses as derived from pharmacy data may exceed the recommended doses indicated in the summary of product characteristics.

Given the importance of treatment persistence in maintaining glycemic control over time and reducing healthcare costs, these results suggest that dulaglutide may help improve clinical and economic outcomes for patients with T2DM initiating GLP-1 RA treatment. Further studies would be useful to explore the potential clinical and economic benefits associated within the GLP-1 RA class.

\section{ACKNOWLEDGEMENTS}

Funding. Sponsorship for this study and article processing charges was funded by Eli Lilly and Co., Indianapolis, IN, USA.

Authorship. All named authors meet the International Committee of Medical Journal Editors (ICMJE) criteria for authorship for this manuscript, take responsibility for the integrity of the work as a whole and have given final approval to the version to be published. Only QuintilesIMS had full access to the raw anonymized data. All authors had access to the aggregated results of this study and take complete responsibility for the accuracy of the data integrity of the work as a whole and have given final approval to the version to be published. The authors thank Sarah Jenner, employee of QuintilesIMS, who has been involved in the study set up and protocol writing.

Disclosures. Trulicity ${ }^{\circledR}$ (dulaglutide) is a registered trademark owned and licensed by Eli Lilly and Co., its subsidiaries or affiliates. QuintilesIMS received consulting fees from Eli Lilly and Co. for the conduct of this study and the manuscript preparation. Kirsi Norrbacka is an employee of Eli Lilly and Co. Giovanni Biricolti is an employee of Eli Lilly and Co. Serena Losi is an employee of Eli Lilly and Co. Jeremie Lebrec is an employee of Eli Lilly and Co. Marco Orsini Federici is an employee of Eli Lilly and Co. Janette McQuillan is an employee of QuintilesIMS. Catrina Richards is an employee of QuintilesIMS. Cristiana Miglio is an employee of QuintilesIMS.

Compliance with Ethics Guidelines. This study involved a retrospective cohort analysis with secondary use of pseudo-anonymized data from the Italian LRx database, and the analysis does not contain new studies with human or animal subjects performed by any of the authors. Research ethics approval was not required.

Data Availability. The data sets generated during and/or analyzed during the current 
study are not publicly available because of patient confidentiality restrictions but are available from the corresponding author on reasonable request.

Open Access. This article is distributed under the terms of the Creative Commons Attribution-NonCommercial 4.0 International License (http://creativecommons.org/licenses/ by-nc/4.0/), which permits any noncommercial use, distribution, and reproduction in any medium, provided you give appropriate credit to the original author(s) and the source, provide a link to the Creative Commons license, and indicate if changes were made.

\section{REFERENCES}

1. Disoteo O, Grimaldi F, Papini E, Attanasio R, Tonutti L, Pellegrini MA, et al. State-of-the-art review on diabetes care in Italy. Ann Glob Health. 2015;81(6):803-13.

2. Marcellusi A, Viti R, Sciattella P, Aimaretti G, De Cosmo S, Provenzano V, et al. Economic aspects in the management of diabetes in Italy. BMJ Open Diabetes Res Care [Internet] BMJ Spec J. 2016;4(1): e000197. https://doi.org/10.1136/bmjdrc-2016-000 197 (cited 11 Oct 2017).

3. Trujillo JM, Nuffer W. GLP-1 Receptor agonists for type 2 diabetes mellitus: recent developments and emerging agents. Pharmacother J Hum Pharmacol Drug Ther [Internet]. 2014;34(11):1174-86. http:// www.ncbi.nlm.nih.gov/pubmed/25382096 (cited 11 Oct 2017).

4. Società Italiana di Diabetologia AMD. Standard italiani per la cura del diabete mellito 2014 [Internet]. 2014. http://www.standarditaliani.it/file/ allegati/STANDARD_2014_May07_15.pdf (cited 11 Oct 2017).

5. Inzucchi SE, Bergenstal RM, Buse JB, Diamant $\mathrm{M}$, Ferrannini E, Nauck M, et al. Management of hyperglycemia in type 2 diabetes, 2015: a patientcentered approach: update to a position statement of the American Diabetes Association and the European Association for the Study of Diabetes. Diabetes Care. 2015;38(1):140-9.

6. Eng C, Kramer CK, Zinman B, Retnakaran R. Glucagon-like peptide-1 receptor agonist and basal insulin combination treatment for the management of type 2 diabetes: a systematic review and meta-analysis. Lancet [Internet]. 2014;384(9961): 2228-34. http://linkinghub.elsevier.com/retrieve/ pii/S0140673614613350 (cited 26 Nov 2016).

7. Prasad-Reddy L, Isaacs D. A clinical review of GLP-1 receptor agonists: efficacy and safety in diabetes and beyond. Drugs Context [Internet]. 2015;4:1-19. http://www.ncbi.nlm.nih.gov/pubmed/26213556 (cited 11 Oct 2017).

8. Giorgino F, Benroubi M, Sun J-H, Zimmermann AG, Pechtner V. Efficacy and safety of once-weekly dulaglutide versus insulin glargine in patients with type 2 diabetes on metformin and glimepiride (AWARD-2). Diabetes Care. 2015;38(12):2241-9.

9. Alatorre C, Fernández Landó L, Yu M, Brown K, Montejano L, Juneau P, et al. Treatment patterns in patients with type 2 diabetes mellitus treated with glucagon-like peptide-1 receptor agonists: Higher adherence and persistence with dulaglutide compared with once-weekly exenatide and liraglutide. Diabetes Obes Metab[Internet]. 2017;19(7):953-61. https://doi.org/10.1111/dom.12902 (cited 2017 Oct 11).

10. Rhee MK, Slocum W, Ziemer DC, Culler SD, Cook $\mathrm{CB}$, El-Kebbi IM, et al. Patient adherence improves glycemic control. Diabetes Educ [Internet]. 2005;31(2):240-50. http://www.ncbi.nlm.nih.gov/ pubmed/15797853 (cited 6 Mar 2017).

11. Lee WC, Balu S, Cobden D, Joshi AV, Pashos CL. Medication adherence and the associated healtheconomic impact among patients with type 2 diabetes mellitus converting to insulin pen therapy: an analysis of third-party managed care claims data. Clin Ther [Internet]. 2006;28(10):1712-25. http:// www.ncbi.nlm.nih.gov/pubmed/17157128 (cited 11 Oct 2017).

12. Stuart BC, Simoni-Wastila L, Zhao L, Lloyd JT, Doshi JA. Increased persistency in medication use by U.S. Medicare beneficiaries with diabetes is associated with lower hospitalization rates and cost savings. Diabetes Care [Internet]. 2009;32(4):647-9. http://www.ncbi.nlm.nih.gov/pubmed/19171724 (cited 11 Oct 2017).

13. Nagrebetsky A, Griffin S, Kinmonth AL, Sutton S, Craven A, Farmer A. Predictors of suboptimal glycaemic control in type 2 diabetes patients: the role of medication adherence and body mass index in the relationship between glycaemia and age. Diabetes Res Clin Pract [Internet]. 2012;96(2):119-28. http://www.ncbi.nlm.nih.gov/pubmed/22261095 (cited 11 Oct 2017).

14. Buysman EK, Liu F, Hammer M, Langer J. Impact of medication adherence and persistence on clinical and economic outcomes in patients with type 2 diabetes treated with liraglutide: a retrospective 
cohort study. Adv Ther [Internet]. 2015;32(4): 341-55. http://www.ncbi.nlm.nih.gov/pubmed/ 25832470 (cited 11 Oct 2017).

15. Lin J, Lingohr-Smith M, Fan T. Real-world medication persistence and outcomes associated with basal insulin and glucagon-like peptide 1 receptor agonist free-dose combination therapy in patients with type 2 diabetes in the US. Clinicoecon Outcomes Res [Internet]. 2017;9:19-29. http://www.ncbi.nlm. nih.gov/pubmed/28053550 (cited 11 Oct 2017).

16. Divino V, DeKoven M, Khan FA, Boye KS, Sapin H, Norrbacka K. GLP-1 RA treatment patterns among type 2 diabetes patients in five European countries. Diabetes Ther [Internet]. 2017. https://doi.org/10. 1007/s13300-016-0224-5 (cited 2017 Feb 2).

17. Divino V, DeKoven M, Hallinan S, Varol N, Wirta $\mathrm{SB}$, Lee WC, et al. Glucagon-like peptide-1 receptor agonist treatment patterns among type 2 diabetes patients in six European countries. Diabetes Ther [Internet]. 2014;5(2):499-520. https://doi.org/10. 1007/s13300-014-0087-6 (cited 2017 Oct 24).

18. McDonell A, Kiiskinen U, Zammit D, Kotchie R, Thuresson P-O, Nicolay C, et al. Estimating the real world daily usage and cost for exenatide twice daily and liraglutide in Germany, the Netherlands, and the UK based on volumes dispensed by pharmacies. Clin Outcomes Res [Internet]. 2015;7:95. http:// www.ncbi.nlm.nih.gov/pubmed/25674008 (cited 11 Oct 2017)

19. Wilke T, Mueller S, Groth A, Berg B, Fuchs A, Sikirica $M$, et al. Non-persistence and non-adherence of patients with type 2 diabetes mellitus in therapy with GLP-1 receptor agonists: a retrospective analysis. Diabetes Ther [Internet]. 2016; 7(1):105-24. http://www.ncbi.nlm.nih.gov/ pubmed/26695499 (cited 4 Nov 2016).

20. Miller L-A, Burudpakdee C, Zagar A, Bhosle M, Reaney M, Schabert VF, et al. Exenatide BID and liraglutide QD treatment patterns among type 2 diabetes patients in Germany. J Med Econ [Internet]. 2012;15(4):746-57. https://doi.org/10.3111/ 13696998.2012.679756 (cited 2017 Oct 11).
21. EMA. Summary of product characteristics [Internet]. http://www.ema.europa.eu/ema/index.jsp? curl=pages/regulation/document_listing/document_ listing_000357.jsp (cited 11 Oct 2017).

22. European Medicines Agency. Summary of product characteristics: dulaglutide [Internet]. 2014. http:// www.ema.europa.eu/docs/en_GB/document_library/ EPAR_-_Product_Information/human/002825/WC50 0179470.pdf (cited 13 Jan 2017).

23. European Medicines Agency. Summary of product characteristics: liraglutide. 2009.http://www.ema. europa.eu/docs/en_GB/document_library/EPAR_Product_Information/human/001026/WC5000500 17.pdf (cited 11 Oct 2017).

24. European Medicines Agency. Summary of product characteristics: exenatide (Byetta). 2006. http:// www.ema.europa.eu/docs/en_GB/document_library/ EPAR_-_Product_Information/human/000698/WC50 0051845.pdf (cited 11 Oct 2017).

25. Lapolla A, Frison V, Bettio M, Pos MD, Rocchini P, Panebianco $G$, et al. Correlation between baseline characteristics and clinical outcomes in a large population of diabetes patients treated with liraglutide in a real-world setting in Italy. Clin Ther [Internet]. 2015;37(3):574-84. http://www.ncbi. nlm.nih.gov/pubmed/25626486 (cited 19 Jan 2018).

26. Lapolla A, Berra C, Boemi M, Bossi AC, Candido R, Di Cianni $G$, et al. Long-term effectiveness of liraglutide for treatment of type 2 diabetes in a reallife setting: a 24-month, multicenter, non-interventional, retrospective study. Adv Ther [Internet]. 2017. https://doi.org/10.1007/s12325-017-0652-2 (cited 2018 Jan 19).

27. Fadini GP, Simioni N, Frison V, Dal Pos M, Bettio M, Rocchini $\mathrm{P}$, et al. Independent glucose and weightreducing effects of Liraglutide in a real-world population of type 2 diabetic outpatients. Acta Diabetol [Internet]. 2013;50(6):943-9. http://www.ncbi.nlm. nih.gov/pubmed/23754673 (cited 19 Jan 2018). 